\title{
LATTICE DEFORMATION STUDIES IN SILICON IMPLANTED WITH HIGH-ENERGY PROTONS
}

\author{
K. Wieteska, K. Dlużewska \\ Institute of Atomic Energy, 05-400 Otwock-Świerk, Poland
}

AND W. WIERZCHOWSKI

Institute of Electronic Materials Technology, Wólczyńska 133, 01-919 Warszawa, Poland

The deformation of crystal lattice in silicon implanted with protons of energy 1.6-9 MeV was studied by means of X-ray topography and double-crystal rocking curve measurements. The samples were investigated as-implanted and after thermal and electron annealing. The surface relief of the implanted part of the crystal was also revealed with optical methods. As-implanted wafers exhibited spherical bending being convex at the implanted side. Thermal and electron annealing caused a dramatic increase in bending of the implanted part while the bending of the remaining part of the sample was reduced. A characteristic behaviour of a double-crystal topographic contrast in the annealed crystals was explained due to bending of the shot-through layer along the Gaussian profile.

PACS numbers: $61.10 .-\mathrm{i}, 61.80 .-\mathrm{x}$

\section{Introduction}

An important effect of the ion implantation in crystals is an introduction of lattice deformation connected with a significant stress level. This effect increases with the energy of the implanted ions and their dose and very often it causes strong bending of the sample. The crystal deformation and the stress level can be very effectively studied by means of X-ray diffraction methods especially those based on double-crystal spectrometers [1-3].

In the present paper the silicon implanted with protons of relatively high energies up to $9 \mathrm{MeV}$ was studied. The investigations were performed for as-implanted crystals as well as after thermal and electron annealing. Some results pointing a significant change of the deformation of the crystals after the annealing processes have been already published [4-5]. 


\section{Experimental}

The implantation was performed in dislocation-free $5 \Omega \mathrm{cm} P$ doped silicon (111) oriented single crystals. The energies of protons were of 1.6 and $9 \mathrm{MeV}$ and the doses from $2 \times 10^{17}$ to $5 \times 10^{17} \mathrm{~cm}^{-2}$. According to Ref. [6] the ion ranges were $35 \mu \mathrm{m}$ and $600 \mu \mathrm{m}$, respectively.

The samples were comparatively studied as-implanted and after thermal and/or electron annealing. When the sole thermal annealing was applied it was performed up to $600^{\circ} \mathrm{C}$ in the steps of $100^{\circ} \mathrm{C}$ during 20 min each. The mixed thermal processing was realised with two periods of 1 and 3 hours thermal annealing up to $400^{\circ} \mathrm{C}$ and several electron beam irradiations with $1.5 \mathrm{MeV}$ electrons up to the dose of $1.8 \times 10^{18} \mathrm{~cm}^{-2}$. In the case of the sole electron beam annealing, the dose of $2.5 \times 10^{18} \mathrm{~cm}^{-2}$ was achieved.

X-ray doublc-crystal topographs were obtained using $\mathrm{Cu} K_{\alpha}$ radiation in $(511,-333),(333,-333)$ and $(111,-111)$ parallel spectrometer settings. The pictures were taken for different regions of the rocking curve and different azimuthal positions of the sample. In some cases the $(444,-444)$ rocking curves were measured using Mo $K_{\alpha_{1}}$ radiation. The samples were also studied with Berg-Barrett method using (111) reflection of $\mathrm{Fe} K_{\alpha}$ and $\mathrm{Cu} K_{\alpha}$ radiation.

The curvature of the surface layer was controlled taking optical photogray, o of the image of the grid reflected by the implanted surface of the sample.

\section{Results and discussion}

The characteristic feature of $9 \mathrm{MeV}$ as-implanted samples was a significant bending of the whole wafer. It may be seen from the representative series of double-crystal topographs shown in Fig. 1. In this case the sample was $1.6 \mathrm{~mm}$ thick. Due to the crystal curvature only a relatively narrow strip remains in the reflecting range at each position at the rocking curve. The radius of curvature evaluated from the width of the strip and its location different for different angle of angular settings was in the range of $30-50 \mathrm{~m}$. The implanted area of $2 \mathrm{~cm}$ diameter did not produce a visible contrast. The shape of the strip in the topographs changed from straight, slightly widened on the boundaries of the sample, into almost closed contours for the position far from the maximum. This behaviour of the contrast points the higher bending in the middle part of the sample while their boundaries remain more flat. The character of the contrast was independent of the azimuthal position of the sample with respect to the diffraction vector indicating the axial symmetry of the crystal deformation. In the present case the parts of the sample closer to the incident beam were reproduced at a smaller glancing angle which points that the sample is convex at the implanted side. The double-crystal image was similar for other side of the sample showing that the contrast is mainly due to the bending.

As it was already reported $[4,5]$ a significant effect of thermal annealing was observed when the temperature of $600^{\circ} \mathrm{C}$ was exceeded. The similar effect was produced by electron beam annealing. The character of the changes is shown in Fig. 2. It may be noticed that in the vicinity of the maximum of the rocking curve a non-implanted part of the sample reflects the X-ray beam in an angular range 

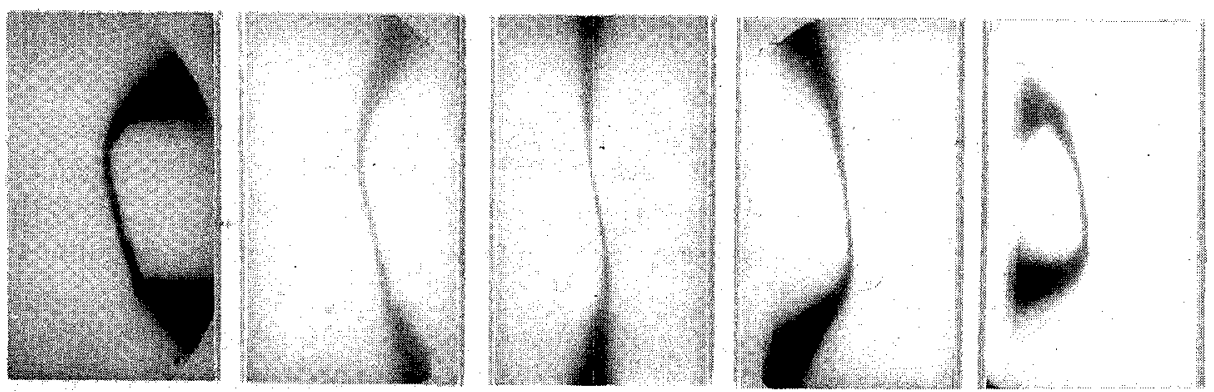

Fig. 1. A series of five $(511,-333)$ double-crystal topographs in $\mathrm{Cu} K_{\alpha}$ radiation of $1.6 \mathrm{~mm}$ thick silicon wafer as-implanted with $9 \mathrm{MeV}$ protons for angular settings changed stepwise by 3 arcsec.

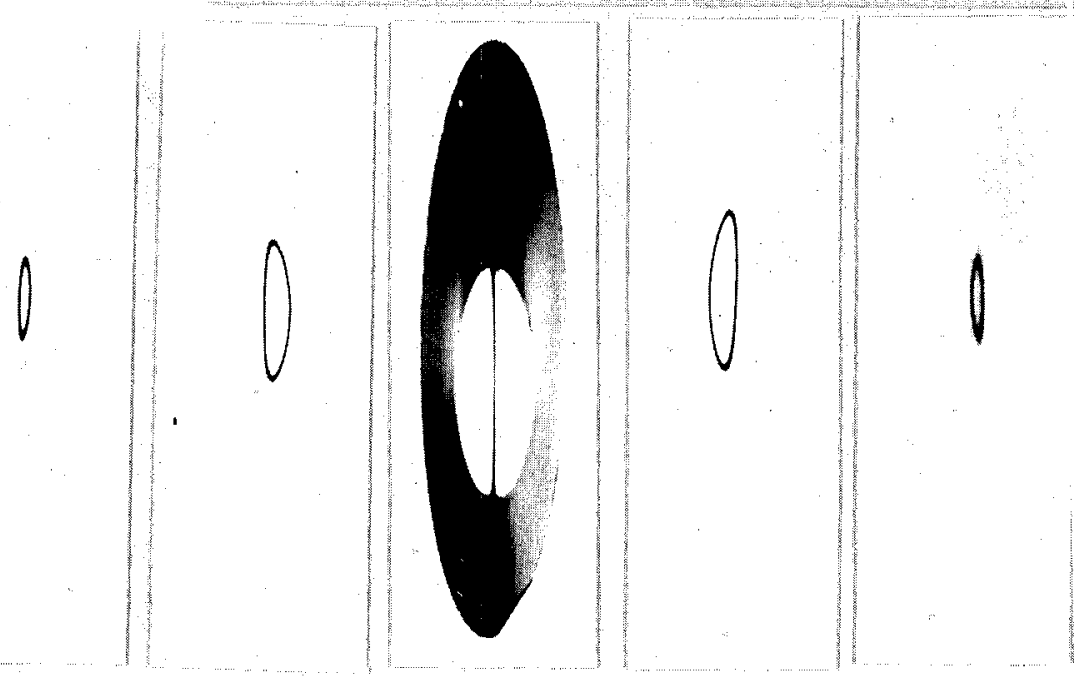

Fig. 2. A series of $(111,-111) \mathrm{Cu} K_{\alpha}$ double-crystal topographs of the sample annealed up to $600^{\circ} \mathrm{C}$. The angular positions are changed by 2 arcminutes.

not very much wider than for the ideal crystal. A dramatic change is observed in the implanted area. Close to the maximum a very narrow strip passing through the middle of the implanted region is reflecting together with the non-implanted region. Further from the maximum we observe a very narrow and closed bean shaped contour. The diameter of this contour decreases with the angular distance from the maximum and the areas situated closer to the incident beam are reproduced for smaller angular settings.

This characteristic behaviour of the double-crystal images suggests that the deformation changed from the bending of the whole sample to the bending of the shot-through layer mainly. This was proved by a kind of a contrast simulation where the contrast was approximated by a contour of an equal change of the reflecting condition assuming the deformation of the surface layer along the Gaussian profile. This means that we assume the reflecting planes to be parallel to the 
function

$$
u(r)=A_{1} \exp \left(-\frac{r^{2}}{2 \sigma^{2}}\right),
$$

where $r$ is the radial coordinate, $\sigma$ is the width of the Gaussian profile and $A_{1}$ is a proportional factor. From that assumption we may write the change of the reflection condition $\Delta \Theta_{\perp}$ due to the change of orientation as a function of the radius $r$ and the angular coordinate $\varphi$ measured from the normal to the plane of diffraction

$$
\Delta \Theta_{\perp}(r, \varphi)=\frac{\partial u(r)}{\partial r} \cos \varphi=-A_{1} \exp \left(-\frac{r^{2}}{2 \sigma^{2}}\right)\left(\frac{r}{\sigma^{2}}\right) \cos \varphi .
$$

The bending of the lattice planes causes also the change of the Bragg condition due to the change of interplanar distances which is also of radial symmetry. It is proportional to the local curvature which is proportional to the second derivative of $u(r)$

$$
\frac{\Delta d(r)}{d}=A_{2}\left(-\frac{1}{\sigma^{2}}+\frac{r^{2}}{\sigma^{4}}\right) \exp \left(-\frac{r^{2}}{2 \sigma^{2}}\right),
$$

where $A_{2}$ is also a proportional factor. The total change of the Bragg condition is

$$
\begin{aligned}
& \Delta \Theta_{\mathrm{ef}}(r, \varphi)=\Delta \Theta_{\perp}(r, \varphi)+\frac{\Delta d(r)}{d} \tan \Theta \\
& =\frac{1}{\sigma^{2}}\left(-A_{1} r \cos \varphi-A_{2} \tan \Theta+\frac{A_{2} r^{2}}{\sigma^{2}} \tan \Theta\right) \exp \left(-\frac{r^{2}}{2 \sigma^{2}}\right) .
\end{aligned}
$$

The contours of the effective Bragg condition change coming from (4) for two different ratios of $A_{1} / A_{2}$ are shown in Fig. 3. They are in very good qualitative agreement with the character of the contrast observed in the topographs. The dependence on the ratio $A_{1} / A_{2}$ is not very significant for the situation corresponding to the elastic bend of the plate of the thickness equal to the shot-through layer. If a greater value of parameter $A_{2}$ is assumed, the asymmetry of bean shaped contours for the corresponding negative and positive angular settings is observed. The sole radial distribution of the lattice parameter change would result in concentric contours similarly as in the well-known case of the contrast due to the striation fringes.

The curvature of the surface layer is also visible in the optical photograph of the grid shown in Fig. 4. It should be noticed that the picture shows the virtual image of the grid hence the convex shape of the surface results in a decrease in the grid spacing in the implanted area. From this picture it was possible to evaluate the macroscopic change of the sample thickness of about $5 \mu \mathrm{m}$.

The above results can be explained assuming that the increase in the lattice parameter caused the elastic bending in as-implanted samples. The dramatic change in the deformation in annealed samples is due to destroying the lattice continuity in the mostly damaged layer allowing the bulge of the elastically bent shot-through layer with a larger lattice parameter. It was previously found $[4,5]$ that approaching $600^{\circ} \mathrm{C}$ coincides with the formation of the silane precipitates but the mechanism of the destroying of the lattice continuity is not well understood. It may be connected with some plastic deformation. 

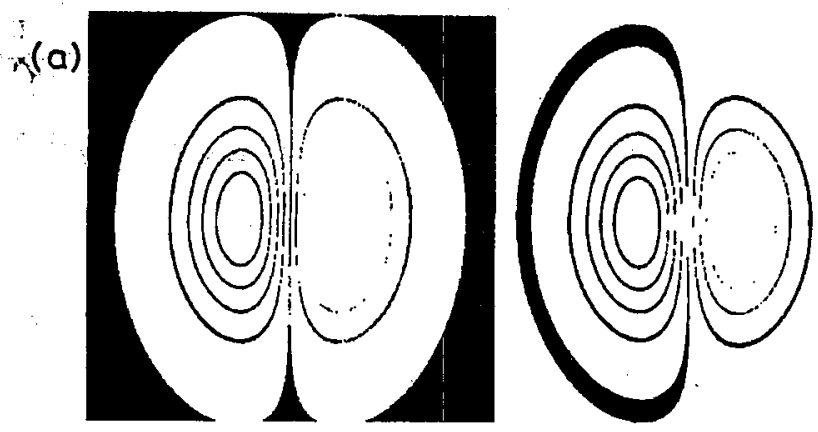

(b)

Fig. 3. The simulation of qualitative character of double-crystal contrast corresponding to bending of shot-through layer along the Gaussian profile. The fringes correspond to a certain narrow region of reflecting range close to different values of the assumed Bragg condition change revealed with the different grey tones: (a) simulation corresponding to the negligible contribution of the lattice parameter change, (b) simulation for the situation where the lattice parameter change causes the asymmetry of the pattern.

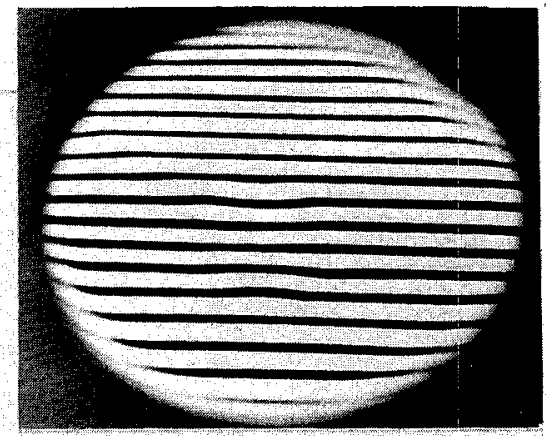

Fig. 4

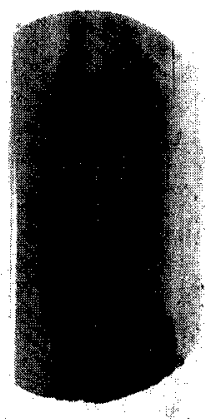

Fig. 5

Fig. 4. The optical photograph of the grid in the beam reflected by the sample shown in Fig. 2. The bending of the surface of the implanted area causes the curvature of the images of the wires seen in the central part of the picture.

Fig. 5. The Berg-Barrett topograph, (111) reflection for $\mathrm{Cu} K_{\alpha}$ radiation, of the sample shown in Fig. 2. Black contrasts correspond to the implanted region boundary with a significant lattice curvature.

The Berg-Barrett topographs of the annealed samples revealed some contrasts on the boundaries of the implanted region as it is shown in Fig. 5. This contrast can be explained due to the most intense lattice bending in these regions.

The exact evaluation of the lattice parameter change due to the ion implantation was possible for smaller energies of ions resulting a smaller ion range. The measurements were performed for the sample implanted with $1.6 \mathrm{MeV}$ protons and 


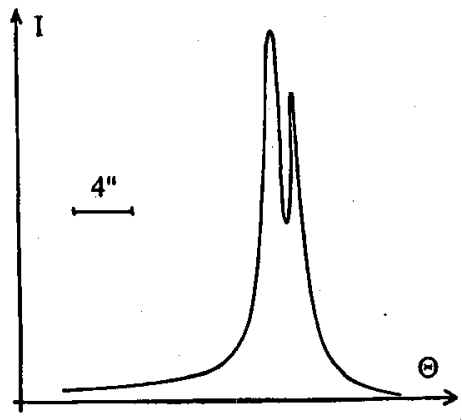

Fig. 6. The rocking curve taken for $(444,-444)$ setting, Mo $K_{\alpha_{1}}$ radiation, of proton implanted crystal of energy $1.6 \mathrm{MeV}$ and the dose $2 \times 10^{17} \mathrm{~cm}^{-2}$. The lower angle peak corresponds to the shot-through layer while the other to the bulk material.

similar ion dose. The representative $(444,-444)$ rocking curve for Mo $K_{\alpha_{1}}$ radiation is shown in Fig. 6. The relative lattice parameter change in the shot-through layer evaluated from the distance between two maxima is equal to $1.25 \times 10^{-5}$.

\section{References}

[1] R. Bubáková, Z. Szmid, Phys. Status Solidi 8, 105 (1965).

[2] M. Lefeld-Sosnowska, E. Zieliniska-Rohoziniska, J. Gronkowski, in: Proc. Int. Conf. Ion Implantation in Semiconductors, Lublin 1974, Inst. Nucl. Res., S'wierk 1974, p. 281.

[3] J. Kub, Phys. Status Solidi A 96, 249 (1986).

[1] J. Tatarkiewicz, K. Wieteska, Phys. Status Solidi A 66, K101 (1981).

[5] K. Biskupska, J. Tatarkiewicz, K. Wieteska, Phys. Status Solidi A 79, K13 (1983).

[6] J. Tatarkiewicz, Phys. Status Solidi A 63, 423 (1980). 